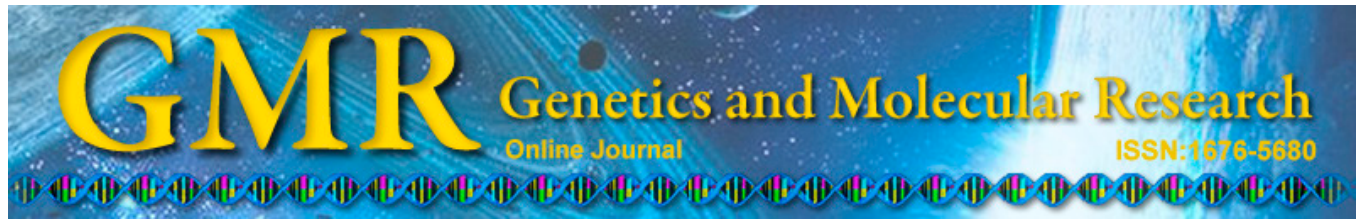

\title{
Molecular cloning and tissue distribution profiles of the chicken $R$-spondin 1 gene
}

\author{
Y.Q. Han ${ }^{1 *}$, J. Geng ${ }^{1 *}$, H.T. Shi ${ }^{2}$, X.M. Zhang ${ }^{1}$, L.L. Du ${ }^{1}$, F.T. Liu ${ }^{1}$, \\ M.M. Li ${ }^{1}$, X.T. Wang ${ }^{1}$, Y.Y. Wang ${ }^{1}$ and G.Y. Yang ${ }^{1}$ \\ ${ }^{1}$ Key Laboratory of Animal Biochemistry and Nutrition, \\ Henan Agricultural University, Ministry of Agriculture, Zhengzhou, \\ Henan Province, China \\ ${ }^{2}$ Department of Veterinary Medicine, \\ Henan University of Animal Husbandry and Economy, Zhengzhou, Henan \\ Province, China \\ *These authors contributed equally to this study. \\ Corresponding authors: Y.Y. Wang / G.Y. Yang \\ E-mail: twgj1@163.com / haubiochem@163.com
}

Genet. Mol. Res. 14 (2): 3090-3097 (2015)

Received May 30, 2014

Accepted September 23, 2014

Published April 10, 2015

DOI http://dx.doi.org/10.4238/2015.April.10.19

\begin{abstract}
Rspol belongs to the Rspo family, which is composed of 4 members (Rspo1-4) that share 40 to $60 \%$ sequence homology and similar domain organizations, and regulate the WNT signaling pathway via a common mechanism. Rspol plays a key role in vertebrate development and is an effective mitogenic factor of gastrointestinal epithelial cells. We report the cloning of chicken Rspol and its gene expression distribution among tissues. It contained an open reading frame of $783 \mathrm{bp}$ encoding a protein of 260 amino acids, and its molecular weight was predicted to be $28.80 \mathrm{kDa}$. Reverse transcriptionpolymerase chain reaction-based gene expression analysis indicated that chicken Rspol was highly expressed in the stomach muscle tissue, but was expressed at low levels in the lung, brain, jejunum, cecum, ileum, spleen, pancreas, kidney, and glandular stomach. These results suggest that Rspo1 plays a major role in muscular immune protection.
\end{abstract}

Key words: $R$-spondin1; Cloning; Tissue distribution; Chicken 


\section{INTRODUCTION}

$R$-spondin1 (Rspo1) belongs to the Rspo family, which is composed of 4 members (Rspo1-4) that share 40 to 60\% sequence homology and similar domain organizations (Kim et al., 2006), and regulate the WNT signaling pathway via a common mechanism (Kim et al., 2008). All four Rspo family members contain a leading N-terminal signal peptide, two furintype cysteine-rich domains, one thrombospondin-type domain, and a C-terminal region rich with positively charged amino acids (Kazanskaya et al., 2004; Kamata et al., 2004). The Rspo family of secreted ligands, similar to canonical WNT family members, activates $\beta$-catenin signaling (Kazanskaya et al., 2004; Kim et al., 2005, 2006; Nam et al., 2006). Expression of Rspo proteins overlaps with expression of WNT ligands during development (Kazanskaya et al., 2004; Nam et al., 2007), indicating that signaling of Rspo and WNT proteins may be closely linked. Rspo genes function as ligands of the orphan receptors - leucine-rich repeat domain-containing G protein-coupled receptor 4 (LGR4) and LGR5 - to regulate WNT/ $\beta$ catenin signaling (Carmon et al., 2011; de Lau et al., 2011). Additionally, the Rspo genes promote the proliferation of cells in the intestinal epithelium (Kim et al., 2005). They are also key-positive regulators of skeletal myogenesis via the $\mathrm{WNT} / \beta$-catenin signaling pathway (Han et al., 2011).

Rspol is a novel regulator of the $\mathrm{WNT} / \beta$-catenin signaling pathway, and its disruption can lead to complete female-to-male sex reversal (Parma et al., 2006; Tomizuka et al., 2008). Rspol is a candidate female sex-determining gene, which participates in suppressing the male pathway in the absence of the sex-determining region of the Y chromosome (SRY) and in maintaining oocyte survival by positively regulating WNT-4 signaling. Rspol is conserved in mammals (human and mouse), birds (chicken), and reptiles (turtle), and all Rspo genes are upregulated during ovarian differentiation (Smith et al., 2008). Furthermore, Rspo1 may promote the proliferation and differentiation of intestinal stem cells and play an important role in the repair process after mucosal damage. Intestinal endocrine cells have high expression of Rspo1, which activates the WNT/ $\beta$-catenin paracrine signaling pathway. Subsequently, the intestinal epithelial stem cells begin to differentiate, proliferate, and repair the damaged intestinal mucosa (Gang, 2009). Rspol itself may protect mice from experimental colitis or from chemotherapy or radiotherapy-induced mucosal injury (Zhao et al., 2007, 2009; Bhanja et al., 2009) and is necessary for normal ductal development in breasts (Chadi et al., 2009). Additionally, Rspo1 plays a key role during vertebrate development (Kim et al., 2005) and an effective mitogenic factor of gastrointestinal epithelial cells. We cloned the cDNA sequence and observed the distribution of Rspol gene expression in chicken tissues.

\section{MATERIAL AND METHODS}

\section{Animals and sample collection}

Gushi chickens (15 days old) were obtained from the animal husbandry station at the Henan Agricultural University. Tissues of the heart, liver, spleen, lung, kidney, muscular stomach, glandular stomach, pancreas, jejunum, rectum, cecum, ileum, duodenum, brain, thymus, and testis were immediately dissected from chickens, frozen in liquid nitrogen, and stored at $-80^{\circ} \mathrm{C}$ until RNA extraction. 


\section{RNA isolation and cDNA synthesis}

Total RNA was extracted from tissues of the heart, liver, spleen, lung, kidney, muscular stomach, glandular stomach, pancreas, jejunum, rectum, cecum, ileum, duodenum, brain, thymus, and testis using a standard Trizol RNA isolation protocol (Invitrogen, Carlsbad, CA, USA). Total RNA was checked by electrophoresis of $1 \mu \mathrm{L}$ RNA on $1.0 \%$ agarose gels stained with ethidium bromide. One microgram of RNA was reverse transcribed with oligo-dT primer (TIANGEN, Beijing, China) and M-MLV reverse transcriptase (Promega, Madison, WI, USA). The cDNA product was stored at $-20^{\circ} \mathrm{C}$.

\section{Amplification of the chicken Rspo1 gene}

Reverse transcription-polymerase chain reaction (RT-PCR) was performed to isolate chicken Rspol using cDNA from the stomach muscle tissue. The primers (5'-ATGCAGCTTGGACTGTTTG-3'; 5'-CTATTGGGCAGGGCTGG-3') used for the amplification of Rspol mRNA were based on the open reading frame (ORF) of the in silico sequence assembly and were determined using the Primer Premier ${ }^{\mathrm{TM}}$ Version 5.0 software (PREMIER Biosoft International, Canada) and checked by BLAST. Each of the primers, including those for the glyceraldehyde-3-phosphate dehydrogenase (GAPDH) gene used as an internal control, and those for the tissue distribution analysis, were synthesized by Shanghai Sango Biological Engineering Technology \& Services Co., Ltd. (Shanghai, China). The 20$\mu \mathrm{L}$ reaction system was composed of the following: $3.0 \mu \mathrm{L}$ cDNA $(100 \mathrm{ng} / \mu \mathrm{L}), 0.5 \mu \mathrm{L} 20$ $\mu \mathrm{M}$ forward primer, $0.5 \mu \mathrm{L} 20 \mu \mathrm{M}$ reverse primer, $10 \mu \mathrm{L}$ Premix Taq DNA polymerase $(1.25$ $\mathrm{U} / 25 \mu \mathrm{L}$; TaKaRa, Dalian, China), and $6.0 \mu \mathrm{L}$ sterile water. PCR was conducted under the following conditions: $95^{\circ} \mathrm{C}$ for $5 \mathrm{~min} ; 30$ cycles of $95^{\circ} \mathrm{C} / 30 \mathrm{~s}, 60^{\circ} \mathrm{C} / 30 \mathrm{~s}$, and $72^{\circ} \mathrm{C} / 50 \mathrm{~s} ; 72^{\circ} \mathrm{C}$ extension for $10 \mathrm{~min}$; and finally a termination step at $14^{\circ} \mathrm{C}$. The $\mathrm{PCR}$ products were analyzed by electrophoresis on a $1 \%$ agarose gel and then visualized with the GelDoc-It ${ }^{\mathrm{TM}}$ Imaging System (Ultra-Violet Products Ltd., UK).

\section{Molecular cloning and sequence analysis of chicken Rspo1}

The PCR products were purified and then cloned into the pMD19-T vector (TaKaRa). The recombinant plasmids were transformed into competent Escherichia coli DH5a using standard molecular techniques. White colonies were checked by PCR, and plasmid DNA was extracted from the positive cultures and verified using restriction enzyme digestion, and the digestion products were analyzed by electrophoresis on a $1 \%$ agarose gel. Colonies with the correct sizes were named pMD19-T-Rspol and five independent plasmid clones were sequenced by Sangon Biotech Co., Ltd. (Shanghai, China). The complete coding sequence of chicken Rspol has been deposited in the GenBank database and was assigned GenBank accession No. KC858867. The cDNA sequence was searched using BLAST and the web servers of the National Center for Biotechnology Information (http://blast.ncbi.nlm.nih.gov/). The protein prediction and analysis were performed using the BioX 2.6 software. 


\section{Tissue distribution of chicken Rspo1}

Expression analyses using RT-PCR were carried out on total RNA extracted from 16 tissues and organs of Gushi chickens. The cDNA from the housekeeping gene GAPDH was used as an internal control. The control primers (5'-TCAAGAAGGGGAACGAGGAC-3'; 5'-TTCTTGGTGCCAGACTTTGC-3') resulted in a 179-bp product. The primers (5'-GGTGGTGCTAAGCGTGTTA-3'; 5'-CCCTCCACAATGCCAA-3') used to perform RTPCR for the tissue distribution analysis were designed and synthesized based on the alignments of the Rspol sequence, resulting in a PCR product of $220 \mathrm{bp}$. The $20-\mu \mathrm{L}$ reaction system was composed of the following: $2.0 \mu \mathrm{L}$ pooled cDNA for each tissue $(100 \mathrm{ng} / \mu \mathrm{L}), 0.5 \mu \mathrm{L} 20 \mu \mathrm{M}$ forward primer, $0.5 \mu \mathrm{L} 20 \mu \mathrm{M}$ reverse primer, $10 \mu \mathrm{L}$ Premix Taq DNA polymerase $(1.25$ $\mathrm{U} / 25 \mu \mathrm{L} ; \mathrm{TaKaRa}$ ), and sterile water (the remaining volume). PCR was conducted under the following conditions: $95^{\circ} \mathrm{C}$ for $5 \mathrm{~min} ; 30$ cycles of $95^{\circ} \mathrm{C} / 30 \mathrm{~s}, 53^{\circ} \mathrm{C} / 30 \mathrm{~s}$, and $72^{\circ} \mathrm{C} / 20 \mathrm{~s} ; 72^{\circ} \mathrm{C}$ extension for $10 \mathrm{~min}$; and a termination step at $14^{\circ} \mathrm{C}$. The RT-PCR products $(10 \mu \mathrm{L})$ from each tissue were electrophoresed on $1.5 \%$ agarose gels, and the Rspol gene expression profiles of Gushi chicken were essentially identical.

\section{RESULTS}

\section{Molecular cloning of chicken Rspo1}

Based on in silico sequence assembly, the Rspol gene was identified from the muscular stomach cDNA library of chickens (Figure 1). It was then isolated, purified, and cloned into the pMD19-T vector (TaKaRa). After transformation and overnight culture, white colonies were confirmed by colony PCR (Figure 2A) and purified pMD19-T-MID1IP1 plasmids of positive colonies were analyzed by digestion with Pst (Figure 2B).

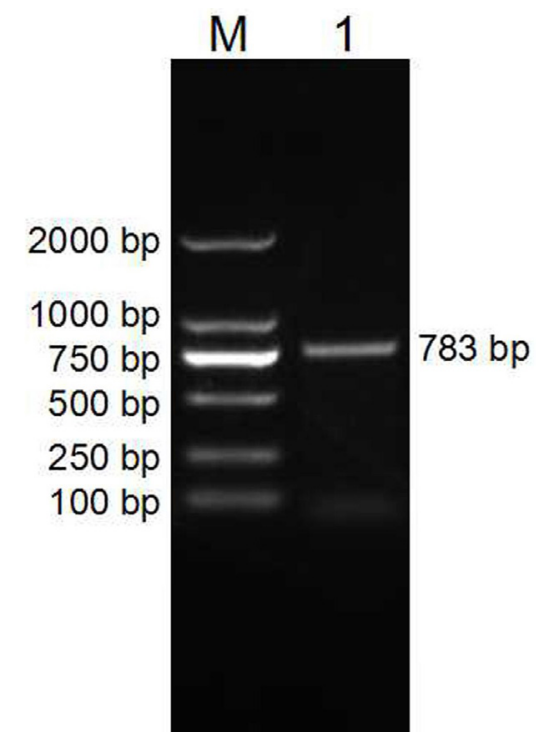

Figure 1. RT-PCR results for chicken Rspo1. Lane M, DL2000 DNA marker; lane 1, PCR product for chicken Rspol. 


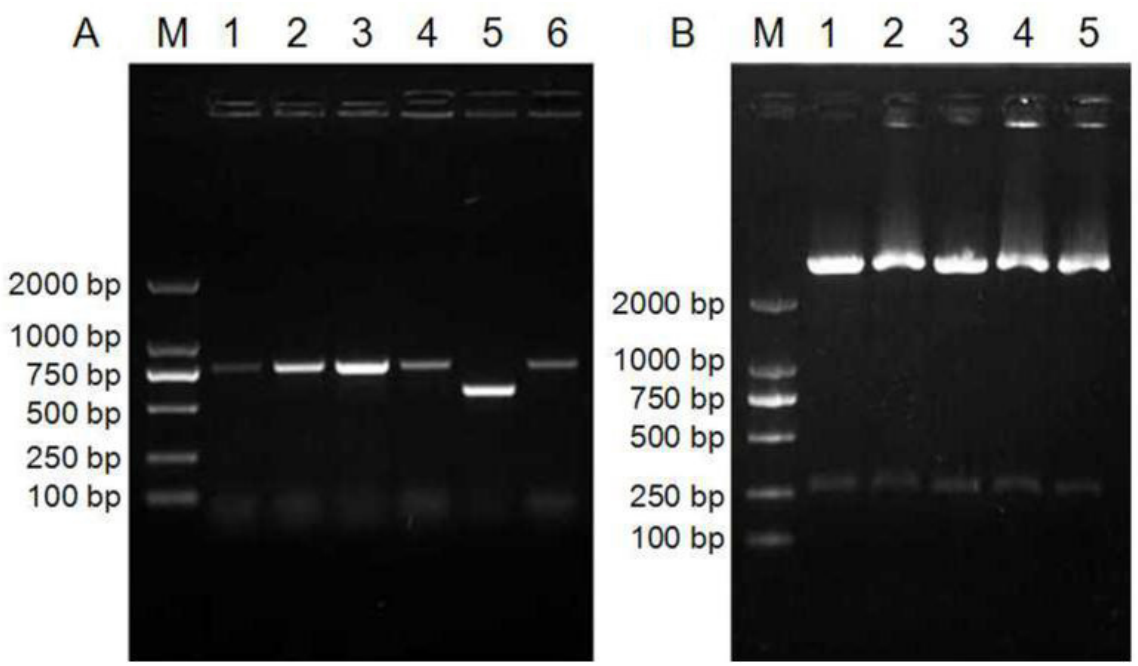

Figure 2. Identification of pMD19-T-Rspol by colony PCR and digestion with Pst. A. The colony PCR identification of pMD19-T-Rspol is shown as follows: Lane M, DL2000 DNA marker; lanes 1-6, PCR results for pMD19-TRspol using amplification primers. B. Digestion-based identification of pMD19-T-Rspo1 is shown as follows: Lane M, DL2000 DNA marker; lanes 1-5, results for digestion of pMD19-T-Rspo1 by Pst.

\section{Sequence analysis}

The ORF of this gene clone was obtained by RT-PCR. The 783-bp ORF was predicted using the BioX 2.6 software; it encoded a 260 -amino acid protein with a putative molecular weight of $28.80 \mathrm{kDa}$, and the isoelectric point was 9.72 . The predicted protein had 48 acidic amino acids and 50 basic amino acids. The nucleotide and predicted amino acid sequences of the ORF of Rspo1 are shown in Figure 3. These cDNA nucleotide sequence analyses revealed that the gene was not homologous to any known chicken genes; the sequence was then submitted to the GenBank database (GenBank accession No. KC858867).

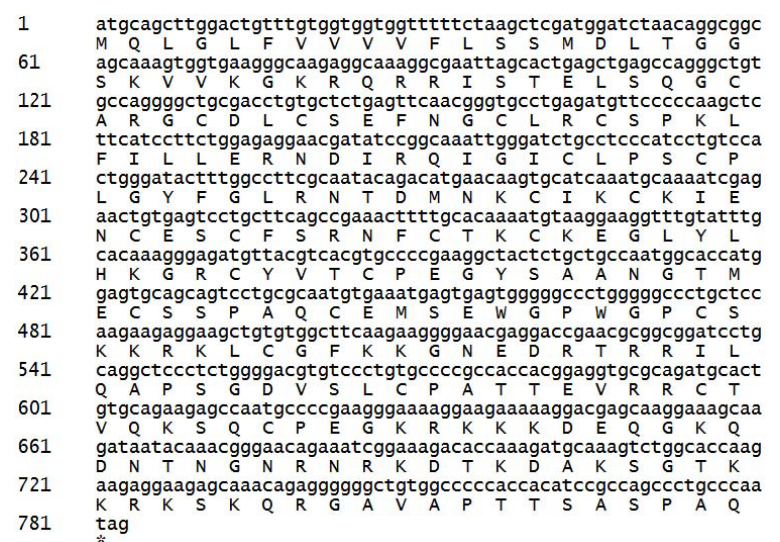

Figure 3. Sequence of the chicken Rspol gene (GenBank accession No. KC858867). The entire deduced amino acid sequence is depicted in single letter code beneath the corresponding nucleotide sequence. The stop codon TAG is shown with an asterisk. 


\section{Tissue distribution of the chicken Rspo1 gene}

The distribution of Rspol gene expression in chicken tissues was analyzed with RTPCR. A total of 16 different tissues from Gushi chickens were included in the study. As shown in Figure 4, the Rspol gene was highly expressed in the stomach muscles, but it was expressed at low levels in the lung, brain, jejunum, cecum, ileum, spleen, pancreas, kidney, glandular stomach, and testis. In other tissues, no band was observed. As an internal control, a GAPDH fragment of $179 \mathrm{bp}$ was amplified in all samples by RT-PCR (Figure 4).

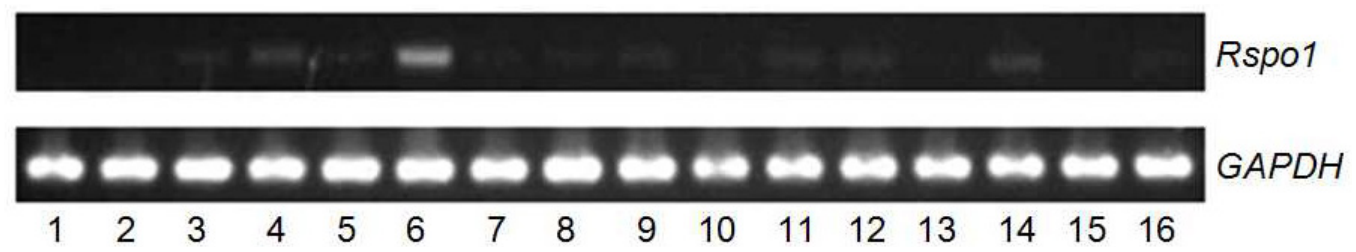

Figure 4. Tissue distribution of chicken Rspol gene expression, determined by RT-PCR analysis. GAPDH expression served as an internal control. The products of Rspol and GAPDH were 220 and $179 \mathrm{bp}$, respectively. The lanes indicate tissue types as follows: 1 , heart; 2 , liver; 3, spleen; 4, lung; 5, kidney; 6 , muscular stomach; 7 , glandular stomach; 8 , pancreas; 9 , jejunum; 10, rectum; 11 , cecum; 12 , ileum; 13 , duodenum; 14 , brain; 15 , thymus; 16 , testis.

\section{DISCUSSION}

We cloned the cDNA of the chicken Rspol gene, which contains a complete ORF of $783 \mathrm{bp}$ encoding a protein of 260 amino acids. Rspol encodes a protein that increases $\mathrm{Wnt} / \beta$-catenin signaling and has pleiotropic functions in development and stem cell growth. Disruption of the human Rspol gene is associated with XX sex reversal, palmoplantar hyperkeratosis, and a predisposition to squamous cell carcinoma of the skin (Parma et al., 2006). In another study, Rspol was demonstrated to be a potent and specific mitogen for the gastrointestinal epithelium, and promotes the proliferation of intestinal crypt cells (Kim et al., 2008). A recent study indicates that Slit2 (a nerve migration factor) and Rspo1 cooperatively induce intestinal stem cells for intestinal homeostasis and repair and significantly prolong overall survival following lethal doses of chemoradiotherapy (Zhou et al., 2013).

Various tissues were collected from chicken for RNA extraction and cDNA synthesis, and were used as templates for RT-PCR analysis. The tissue distribution analysis revealed that the expression of Rspol differs among the tissue types. We only observed significant expression of Rspol in the stomach muscle. In the lung, brain, jejunum, cecum, ileum, spleen, pancreas, kidney, glandular stomach, and testis, Rspol expression is moderate. These data indicated that Rspol, a potent growth factor, markedly increases the efficacy of intestinal cultures (Kim et al., 2005). By contrast, Rspol is ubiquitously expressed in the brain, liver, heart, intestine, kidney, ovary, and testis, with dominant expression in the brain, liver, and ovary in medaka (Zhou et al., 2012). An RNA blot analysis of adult human tissues showed that Rspol is abundantly expressed in the adrenal glands, ovary, testis, thyroid, and trachea, but not in the bone marrow, spinal cord, stomach, leukocytes, colon, small intestine, prostate, thymus, and spleen (Parma et al., 2006). In zebrafish gonads, Rspo1 expression in ovaries is 3.84-fold higher than that in the testes. Rspol is also differentially expressed in kidney and muscle tissue. Weak expression is detected in male hearts, but expression is not detected in female 
hearts, suggesting that Rspol is specifically involved in the development of the male heart (Hang et al., 2011). Taken together, these data indicated that the Rspol gene was differentially expressed among tissues in various organisms, suggesting that it plays a role in various tissues and performs multiple functions, even though the Rspo family is evolutionarily conserved (Kim et al, 2006). However, despite the variation in expression level, Rspol is expressed, without exception, in the brain, kidney, ovary, testis, and intestine of different organisms. Our results showed that the Rspol was mainly expressed in smooth muscle tissues, which indicated that it is expressed in a tissue-specific manner in chickens. Therefore, Rspol may play a major role in muscular immune protection. Because we did not study gene function or protein expression, further studies regarding are required.

\section{ACKNOWLEDGMENTS}

Research supported by a grant from the Ministry of Agriculture (\#2011-G35) and by the Project of National Major Basic Dairy Research “973” Plan (\#2011CB100802).

\section{REFERENCES}

Bhanja P, Saha S, Kabarriti R, Liu L, et al. (2009). Protective role of R-spondin1, an intestinal stem cell growth factor, against radiation-induced gastrointestinal syndrome in mice. PLoS One 4: e8014.

Carmon KS, Gong X, Lin Q, Thomas A, et al. (2011). R-spondins function as ligands of the orphan receptors LGR4 and LGR5 to regulate Wnt/beta-catenin signaling. Proc. Natl. Acad. Sci. USA 108: 11452-11457.

Chadi S, Buscara L, Pechoux C, Costa J, et al. (2009). R-spondin1 is required for normal epithelial morphogenesis during mammary gland development. Biochem. Biophys. Res. Commun. 390: 1040-1043.

de Lau W, Barker N, Low TY, Koo BK, et al. (2011). Lgr5 homologues associate with Wnt receptors and mediate R-spondin signalling. Nature 476: 293-297.

Gang Y (2009). The expression and its mechanism of R-Spondin1 in inteestinal musoca after intestinal ischemiareperfusion. Master's thesis, Medical University, Guangzhou.

Han XH, Jin YR, Seto M and Yoon JK (2011). A WNT/beta-catenin signaling activator, R-spondin, plays positive regulatory roles during skeletal myogenesis. J. Biol. Chem. 286: 10649-10659.

Hang Y, Li F, Sun D, Liu J, et al. (2011). Molecular analysis shows differential expression of R-spondin1 in zebrafish (Danio rerio) gonads. Mol. Biol. Rep. 38: 275-282.

Kamata T, Katsube K, Michikawa M, Yamada M, et al. (2004). R-spondin, a novel gene with thrombospondin type 1 domain, was expressed in the dorsal neural tube and affected in Wnts mutants. Biochem. Biophys. Acta 1676: 51-62.

Kazanskaya O, Glinka A, del Barco Barrantes I, Stannek P, et al. (2004). R-Spondin2 is a secreted activator of Wnt/betacatenin signaling and is required for Xenopus myogenesis. Dev. Cell. 7: 525-534.

Kim KA, Kakitani M, Zhao J, Oshima T, et al. (2005). Mitogenic influence of human R-spondin1 on the intestinal epithelium. Science 309: 1256-1259.

Kim KA, Zhao J, Andarmani S, Kakitani M, et al. (2006). R-Spondin proteins: a novel link to beta-catenin activation. Cell Cycle 5: 23-26.

Kim KA, Wagle M, Tran K, Zhan X, et al. (2008). R-Spondin family members regulate the Wnt pathway by a common mechanism. Mol. Biol. Cell. 19: 2588-2596.

Nam JS, Turcotte TJ, Smith PF, Choi S, et al. (2006). Mouse cristin/R-spondin family proteins are novel ligands for the Frizzled 8 and LRP6 receptors and activate beta-catenin-dependent gene expression. J. Biol. Chem. 281: 13247-13257.

Nam JS, Turcotte TJ and Yoon JK (2007). Dynamic expression of R-spondin family genes in mouse development. Gene Expr. Patterns 7: 306-312.

Parma P, Radi O, Vidal V, Chaboissier MC, et al. (2006). R-spondin1 is essential in sex determination, skin differentiation and malignancy. Nat. Genet. 38: 1304-1309.

Smith CA, Shoemaker CM, Roeszler KN, Queen J, et al. (2008). Cloning and expression of R-Spondin1 in different vertebrates suggests a conserved role in ovarian development. BMC Dev. Biol. 8: 72.

Tomizuka K, Horikoshi K, Kitada R, Sugawara Y, et al. (2008). R-spondin1 plays an essential role in ovarian development through positively regulating Wnt-4 signaling. Hum. Mol. Genet. 17: 1278-1291. 
Zhao J, de Vera J, Narushima S, Beck EX, et al. (2007). R-spondin1, a novel intestinotrophic mitogen, ameliorates experimental colitis in mice. Gastroenterology 132: 1331-43.

Zhao J, Kim KA, De Vera J, Palencia S, et al. (2009). R-Spondin1 protects mice from chemotherapy or radiation-induced oral mucositis through the canonical Wnt/beta-catenin pathway. Proc. Natl. Acad. Sci. U. S. A. 106: 2331-2336.

Zhou L, Charkraborty T, Yu X, Wu L, et al. (2012). R-spondins are involved in the ovarian differentiation in a teleost, medaka (Oryzias latipes). BMC Dev. Biol. 12: 36.

Zhou WJ, Geng ZH, Spence JR and Geng JG (2013). Induction of intestinal stem cells by R-spondin 1 and Slit2 augments chemoradioprotection. Nature 501: 107-111. 